\title{
Implementation of Enterprise Resource Planning (ERP) in Bangladesh -Opportunities and Challenges
}

\author{
Md Shahnur Azad Chowdhury ${ }^{1}$, Mohammad Toufiqur Rahman ${ }^{1}$, A. M. Shahabuddin ${ }^{1}$, Md Rizwan $\operatorname{Hassan}^{1}$ \& \\ Mohammad Shyfur Rahman Chowdhury ${ }^{2}$ \\ ${ }^{1}$ Associate Professor, Department of Business Administration, International Islamic University Chittagong, \\ Bangladesh \\ 2 Assistant Professor, Department of Business Administration, International Islamic University Chittagong, \\ Bangladesh \\ Correspondence: Md Shahnur Azad Chowdhury, Department of Business Administration, International Islamic \\ University Chittagong, Bangladesh. E-mail: tipu_iiuc@yahoo.com
}

Received: August 4, 2021

Accepted: September 10, 2021

Online Published: September 18, 2021

doi:10.5539/ijbm.v16n11p1

URL: https://doi.org/10.5539/ijbm.v16n11p1

\begin{abstract}
An enterprise resource planning (ERP) project is a sizable enterprise application either in the business and engineering perspective. It creates huge alterations and frequently entails calling into question several traditional roles within the business, for which individuals are rarely well ready. It frequently involves the execution of numerous new approaches and their integration with existing programs and frequently reflects one of the bigger IT jobs -- one of the highly crucial ones in many businesses, cooperation between industry and IT extends into international cooperation between many small business units and many entities of their IT division. The achievement of this undertaking will count on the caliber of the cooperation. Before beginning such a difficult endeavor, certain conditions have to be ensured. The business has to see to adulthood in ERP and also the management and technical capacities of their IT division and the capability of the business and IT to work together. Without these requirements, companies wouldn't have the ability to lead and get different stages of an ERP project. This preparation could require a few educational and communication efforts which can be essential for success. This study has been undertaken to address the viability of installing ERP in any enterprise and its benefits and challenges in Bangladesh. ERPs are highly technical issues with numerous benefits. It requires a lot of preconditions to be installed. By reviewing the literature and going through several recent research, a concept is tried to be developed here in this study. Idea about current status of ERP practices in Bangladesh has been generated from various circulars and reports produced by the companies. The study found that productivity and profits are increased by implementing ERP in the local enterprises but a lot of challenges are to be addressed. The infrastructure of IT and power supply should be ensured to gain optimum advantages. The employees should be well trained to run the system successfully.
\end{abstract}

Keywords: Enterprise Resource Planning (ERP), opportunities, challenge, productivity

\section{Introduction}

ERP stands for Enterprise Resource Planning. ERP software is comprised of powerful and strategic business process management tools that can be used to manage information within an organization. While every company and organization operating today is different, they all face a common challenge: in order to stay competitive in today's business environment, they need a dependable and efficient way to store and access information. That's where ERP systems come into play. ERP systems integrate all facets of an enterprise into one comprehensive information system that can be accessed by individuals across an entire organization. Enterprise Resource Planning (ERP) systems are company computer automation aids that enable businesses to digitize business activities and discuss information and practices throughout the business and create and access real-time information. The most important aim of ERP systems would be to incorporate all units on a single computer system that serves each of the organization's requirement. ERP structures are frequently employed by businesses of many scopes and functions since their prospective gains are too important to dismiss. To be a leader in the contemporary commercial arena, companies need to establish decent business practices and efficiently share data internally through their providers, partners, and clients. 


\subsection{Objectives}

The objectives of the study are given below:

1. To assess the scopes of ERP implementation in Business firms of Bangladesh.

2. Find out the problems during implementation.

3. To recommend suggestions to overcome the problems.

\subsection{Literature Review}

Bahssas (2015) came up with a number of some of the capable new ERP evolutionary design and models that could contribute to ERP design and extend new market for the potential customers. AlBar D., A., M., in 2014 discussed the opportunities, challenges, emergence, implementation and importance of using ERP inside the organization. Rahad (2014) proposed a new method for accessing ERP system regarding with foreign network. Kabir (2020) evaluated the impact of Enterprise Resources Planning (ERP) implementation on the productivity and profitability of BSRM Steels Limited. The study reveals that both the productivity and profitability of BSRM have significantly improved after ERP implementation. Bagranoff and Brewer (2003) conducted a case study to conclude that implementation of the ERP could save an estimated \$30 million annually for the company by reducing unnecessary inventor. Olhager and Selldin (2003) surveyed the use of ERP systems by Swedish manufacturing firms. they concluded that ERP maturity is high in those firms. Even though the ERP system does not reduce information technology costs, the system rapidly improved the availability \& quality of information and integration \& interactions throughout the firm. Saharia Koch and Tucker (2008) conducted a study with an outcome that the internal audit department fulfilled their relevant ERP skills requirement by providing staff with in-house training instead of outsourcing the training programs. Rao and Rao (2009) have reported that information technology, corporate processes, and information exchange significantly affect product turnover efficiency. According to Kallunki, Laitinen, and Silvola (2011) application of ERP system leads to improved financial returns and management control system help the firm achieve future performance goals than informal types. Hunton, Lippincott, and Reck (2003) in their study commented that the ERP adoption helps the firm achieve a competitive advantage over non-ERP adopters. Poston and Grabski (2001) concluded in their research that the firm's performance improved significantly as there was a decrease in the cost of goods sold to revenue ratio. Argote, McEvily and Reagans (2003) highlighted in their study the mechanics of knowledge management and how these mechanisms impact a unit's capacity to create, maintain, and transfer knowledge. Srinivas Nowduri (2003) explained and examined the part of MIS in light of its capacity for choice making. Decision making procedure and its effect on high-level direction in a company organization is described as an emphasis on automatic decision making. Kopáková and Škrobáčková (2006) revealed that engineered conclusions are routine or repetitive and maybe solved via straightforward mechanical processes, like implementing the rules to locate the very ideal solution. As much as $90 \%$ of management choices are all programmed. Ball (2001) mentioned that within the past couple of decades there's been substantial growth in the number of businesses collecting, preserving, tracking, and assessing information about their abilities with the usage of Human Resource Information Systems (HRIS) applications or other varieties of applications including HRIS performance. Kovach, et al. (2002) described HRIS denotes a systematic process for collecting, preserving, and retrieving information needed by an organization in their assets, personnel actions, and organizational traits. Parry (2010) showed that an integrated HRIS might have a vast assortment of usages from easy spreadsheets to complicated calculations done readily.

ERP brings a lot of benefits to firms as:

1. Integrating the function of a business

2. Real-time information is quickly accessed

3. Uniformity is ensured in information system maintenance

4. Consistency of business functions are supported

\subsection{Enterprise Resource Planning}

ERP systems, in contrast to operating areas, comprise a number of distinct units that provide support. Incorporated within the database are modules for such areas as customer affiliation management, fiscal accounting, SCM (Supply Chain Management), and individual assets. These modules access the database to obtain precise information about their respective areas. Businesses using ERP solutions may choose which modules they want to install based on their requirements, and the modularity of these systems allows them to begin utilizing certain portions of the ERP system in conjunction with other systems in order to save money. A 
typical ERP process is expensive and time-consuming to implement in its entirety; nevertheless, the benefits of comprehensive integration of information throughout the company's different operating areas outweigh the costs and time commitment.

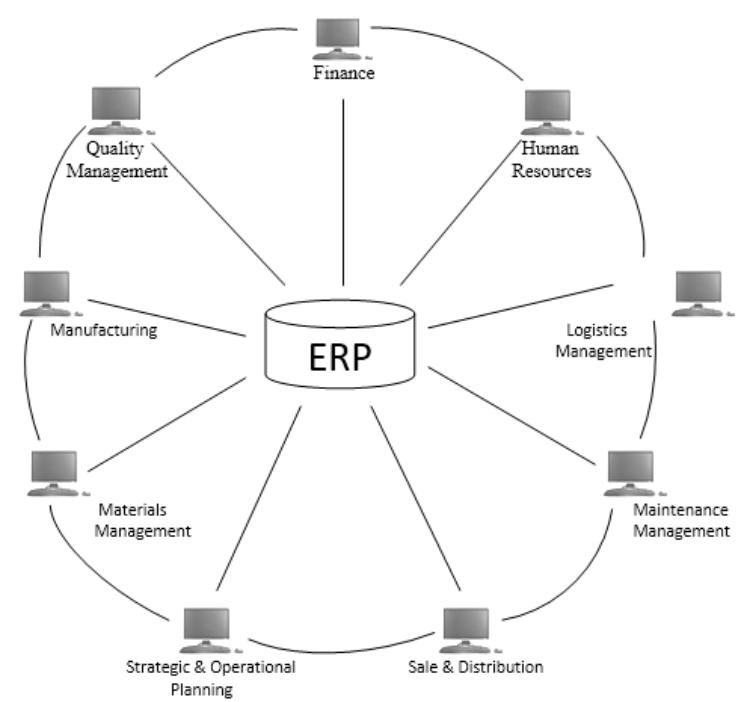

Figure 1. Information integration through ERP systems

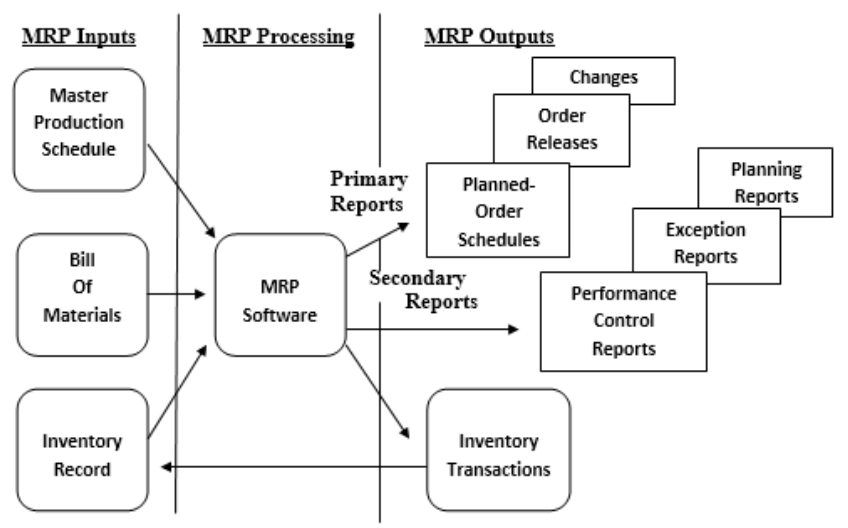

Figure 2. The function of MRP software

From the 1980s forward, MRP-II (Manufacturing Resource Planning) systems began to appear. The MRP-II concept was based on the MRP techniques, but new qualities were added to make it more comprehensive. The techniques focused on the quality of the products, and they were able to schedule and track the implementation of their production strategy. Later, the emphasis of MRP-II programs turned more toward meeting the needs of clients, and the software's capacity to change production processes in order to meet specific client requirements on a timely basis has been tightly regulated by the program. ERP systems were developed in the late 1990s to encompass all of the data flows within a company, and the concepts of e-business and enhanced data sharing across enterprises' data systems were incorporated into the ERP concept in the late 1990s. While Enterprise Resource Planning (ERP) systems were initially designed for manufacturing companies, their use is no longer restricted to a single kind of business or organization, and ERP systems are now being used by a wide range of enterprises and organizations. Service firms are increasingly relying on enterprise resource planning (ERP) systems to improve their decision-making and efficiency. Manufacturing firms are still employing ERPs, but they are now depending on them to handle the whole process associated with a client purchase from beginning to end. Regardless of whether a company is small, medium-sized, or large, modern ERP systems are capable of performing a wide range of activities and duties for diverse departments.

As firms are creating systems to maximize their actions, the demand for incorporations between unlike data 
systems develops. Now ERP systems stay expected to assist in governing company networks. Here figure exemplifies how companies' applications and functionalities have emerged.

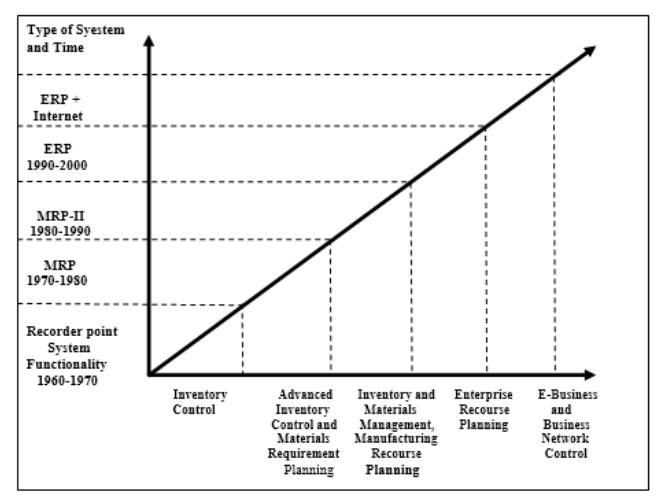

Figure 3. ERP history and the evolution of ERP functionalities

\subsection{ERP Function and Advantages}

According to Statistics Finland (2011), 75 percent of Finnish firms with more than 100 employees use enterprise resource planning systems (ERP systems). Employers with 50-99 employees have a 56 percent adoption rate in this category. Given these data, it is reasonable to assume that adopting an ERP system is advantageous for organizations. From a business perspective, the following is a list of common ERP advantages.

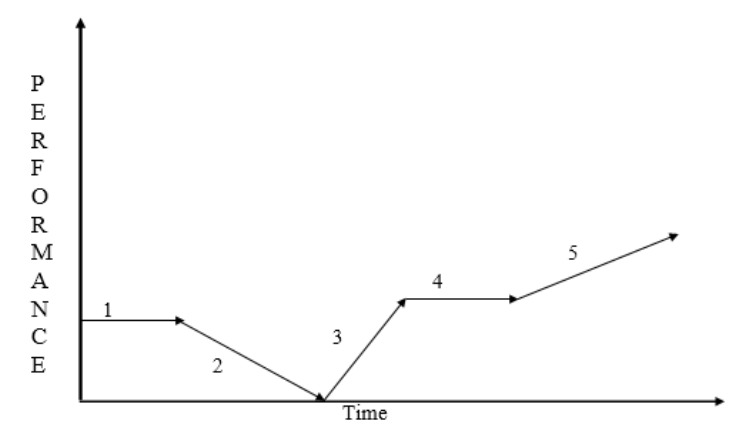

Figure 4. Before, during, and after the deployment of an ERP system, how well a company performs

1: Before ERP Implementation.

2 \& 3: During Implementation.

4: After Implementation, during training

5. After training and going live completely

However, it should be emphasized that the efficiency of an ERP system is only proportional to the degree to which its users cooperate with one another. Another way to phrase it is that the success or failure of an ERP program is determined by the manner in which its users interact with it.

There are a multitude of ways in which an Enterprise Resource Planning system (ERP) may be useful to a company. Consequently, it is difficult to attribute each of the benefits to the machine in a clear manner. ERPs have the potential to boost revenues and decrease expenditures in ways that are difficult to measure. A few of the edges, on the other hand, could not show until a considerable time after the first execution, making them difficult to detect during the brief run.

As can be seen from the figure number 1.4, the operation of a firm will deteriorate as a result of the execution. This reduction can be attributed to the difficulties that employees regularly have in adapting to new means of communication and new corporate communication methods. Workers who are required to carry out their regular duties while participating in the implementation project may get distracted as a result of the project's implementation. Although the decline in this organizational operation is only a short stage, it is possible that the firm will rapidly become more successful than it was before the ERP installation provided the new system is 
properly implemented and utilized.

The integration of data across the distribution chain is, without a question, the most significant advantage of an ERP system, as it has the potential to enhance operational efficiency while also reducing costs and inventories. As an example, the capacity to swiftly access stock levels, product information, client credit histories, and past purchase histories through order input has increased the efficiency of inventory management.

Working with data systems that do not "communicate" with one another is made easier when using a well-functioning ERP system. Information shared across various business objectives is precisely the same across the firm with ERP systems, but information shared between different departments with non-integrated systems may have varying meanings is the case with non-integrated systems. ERP (Enterprise Resource Planning) solutions are becoming increasingly prevalent.

Because the ERP option requires just a single entry of information into the system, the information will be readily available to anybody who uses a computer from that point forward. This removes the need for wasteful manual operations such as repeatedly typing the same precise data into a computer.

ERP systems are typically linked with high prices; however, one advantage of utilizing an ERP system is that data technology maintenance expenses as well as permit fees may be reduced when compared to the costs associated with the use of several different systems. Different techniques must be maintained individually, which is expensive, time-consuming, and complex. The yearly permit fees might become prohibitively expensive, despite the fact that each individual system is charged just a little fee.

It is the primary motive for the existence of businesses in the first place to generate profits. To be expected, after a business has made a big investment in technology, such as in the implementation of an ERP platform, they would seek information on how to increase profitability throughout the computer system. As we all know, the only way to increase profits is to either decrease costs or increase revenues. The ERP program makes a major contribution to improved data quality in a number of dimensions such as accessibility, accuracy, and timeliness, among other things.

Improvements in the quality of advice have an impact on a variety of aspects of an organization, including decision-making procedures and operational operations, among other things. The fact that an ERP can give each of these benefits makes it simpler to accomplish improvements in organizational growth, which should translate to higher profit margins in the long run. When businesses have access to better information, they may operate with less resources, such as less physical labor or machinery, while also boosting revenues through precise sales forecasting and considerably enhanced client service, both of which result in increased profitability.

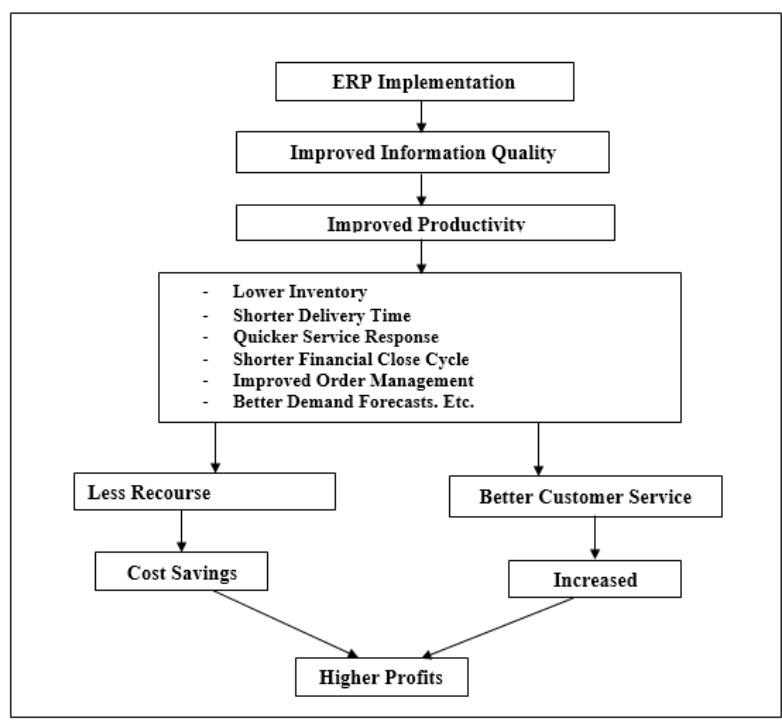

Figure 5. Realization of profits through ERP 


\subsection{Disadvantages of ERP}

Despite the fact that ERP systems may be highly useful to the businesses who employ them, the implementation of those systems has, as previously said, usually proven to be dubious. There are a variety of disadvantages connected with ERP alternatives to consider in addition to worries about the ERP implementation process. The high cost of ERP systems is, without a question, the most major disadvantage of modern technology. The entire cost of this implementation project can be quite costly, and ERP systems are frequently finished late or over budget, increasing the likelihood of failure. The implementation of an enterprise resource planning system is a multimillion-euro project for big businesses, with consulting costs often exceeding the amount of the original ERP permit price. When comparing small and midsize businesses to giant companies, ERP installation costs are lower for small and midsize businesses, but the expenses will be significant for any company that decides to use an ERP system in its operations. Even after the high-cost execution,The systems are frequently prohibitively expensive to maintain and operate. ERP systems exhibit "best practices," which are procedures that are commonly believed to be the most effective ways to carry out certain activities in a given circumstance, as opposed to "best practices" that are not displayed. Despite the fact that best practices can assist employers in improving their company operations, they can also present barriers for ERP deployments because the system imposes regulations on the businesses that use it. The typical processes contained in ERP systems replace old processes, which very frequently enhance the operations of an organization; nevertheless, these new procedures also replace corporate processes that have historically served as a source of competitive advantage in an organization's operations. A particular conventional way of conducting business, for example, may allow for more adaptability and distinction from the competition in some areas where competition is severe and Enterprise Resource Planning (ERP) technologies are frequently utilized. It is possible that using an ERP solution in this situation may result in a loss of competitive edge. The most effective ERP methods may also be incompatible with the business culture, resulting in a "culture clash" and the creation of new challenges.

Businesses must constantly modify their practices in order to fit ERP systems in order to make the most of the best approaches that are incorporated into them. This generally leads in immunity from employees when utilizing the best methods included into ERP systems. Because resistance to change has been identified as a key contributing factor to the failure of information system deployments, it is an issue that has to be addressed and dealt with in the proper manner, as described above. ERP benefits include the reduction of the need for manual labor on the part of the company, which is another aspect that may contribute to user immunity because a number of employees may fear being fired off as a result of the adoption of a new system.

One of the most challenging aspects of ERP is that it may be difficult to tailor it to the particular requirements of individual businesses. In reality, only a tiny number of businesses are able to utilize ERP straight out of the box, and in the majority of situations, it must be modified to fit the unique requirements of the organization. Both the setting of the machine and the alteration of the machine may be considered tailoring in this context. The modification of this program, which may be performed by altering the code of the software, may be very time-consuming and expensive to undertake. When it comes to enterprise resource planning systems, one of the most prevalent criticisms is that they are too rigid and inflexible when it comes to adapting to the unique workflow and business processes of different businesses. Implementation of ERP may result in a lack of flexibility and the necessity for users to reconsider their daily routines under such circumstances.

As previously said, ERP solutions are only as effective as the customers' ability to utilize them effectively. Undoubtedly, some of the applications are complicated and difficult to operate without proper training, which is both time-consuming and expensive.

\subsection{ERP Implementation Project}

The idea of execution is linked with the process of setting up hardware and software on a computer. Specifically, in the context of enterprise resource planning systems, the phrase "execution" refers to the whole project, from the initial project design to installation and training before the machine is actually put into use by the company. The following table summarizes the opinions of the sellers and advisers on the implementation of the transaction. According to the organization's perspective, implementation signifies the beginning of a continuous learning cycle in which the organizational procedures enabled by the ERP are intricately intertwined with the organization's objectives.

ERP installation, like any other activity within a company, necessitates the consideration of concepts such as objectives, deadlines, and funding, as well as scope, tools, stages, tasks, and functions, among other things. ERP installation is similar to any other activity within a company. The concept of aims is the most crucial of all of these concepts to understand. It is critical to note that an ERP installation project is always a business 
improvement project with the goal of improving the overall operational performance of the firm, regardless of the industry. As a consequence, before your project can begin, it is necessary to create business-related objectives and goals for it to be successful. Before beginning the implementation of an ERP system, a company should have a crystal-clear knowledge of why it is doing the project and what the company's goals are that it expects to achieve through the usage of the system before proceeding. Careful planning and preparation may make a major difference in the success of a project's implementation and the achievement of its goals and objectives.

\section{The Effects of Enterprise Resource Planning on Business}

Essentially, enterprise resource planning (ERP) is the unification of many assets in a business or company into a single computer system that meets the needs of all of the company's or organization's numerous branches. The benefits of this include much improved interconnection and communication between divisions, as well as significantly simplified start-to-finish procedures.

\subsection{Task Completion}

One of the most frequently mentioned benefits of business resource planning is the ability to trace products more quickly from manufacture to end-user. When it comes to bespoke computers, Dell Computer, for example, uses an internet-based ordering method. With the help of an ERP, a customer may make an order and pay for the product, and then the order is transferred directly to creation, where the computer is built and shipped. This can be as basic as a trail or a conveyor belt that involves only a small number of people and a single ordering mechanism that all branches have access to, for example.

\subsection{Communication Barriers}

Enterprise resource planning (ERP) eliminates the traditional barriers that exist between departments within a company. For example, the sales division was only one of several "mini-organizations," while purchasing was another, and sending and receiving was still another. These roadblocks to communication and item delivery are addressed through the use of widely available apps. ERP is a standard phrase used by this company to describe how each component of the organization operates as a whole.

\subsection{Cost of Resources}

One last result of ERP on many organizations is a net decrease in the expense of funds used, in addition to the price of information management utilized to produce the business work. With a single management program, there is no need of special management methods that have to be preserved, so a computer tech may have the ability to keep all the organization's applications without needing to be educated in eight distinct software bundles. Additionally, electricity consumption is diminished because ERP systems may often be server-based or perhaps preserved off-road by another party.

\section{EPR in Bangladesh}

\subsection{Environment for ERP Implementation in Bangladesh}

Even though the execution of the Enterprise Resource Planning system is growing quite quickly, the expansion rate is largely observable in developed nations where the business environment is very competitive with accelerated financial development, where it's a solid maturity in information technologies, and it's a solid management commitment. On the other hand, the expansion of ERP implementation in developing nations is moving upwards, but remains long from the developed countries.

Of the entire ERP market globally; the USA occupies 66 percent; Europe occupies 22 percentages while Asia occupies just 9 percent. On the other hand, the ERP adoption speed is growing in Asia, because of economic growth; growing nations in Asia and Latin America have become major goals of significant ERP vendors. Most small and medium scale businesses are now implementing the systems to realize the operational efficiency of the marketplace. Additionally, many ERP vendors have also personalized their ERP platform to occupy the fast increasingly market within this area. This situation isn't distinct in South East Asia.

For Bangladesh, even though a high number of businesses have implemented or are still in the process of executing Enterprise Systems, there is no reliable source to verify their complete numbers as well as the research failed to recognize some prior study within this circumstance addressing this specific matter.

But from the web site advice of the top ERP vendors and in the regional partners in this area it's likely to conceive the increase of execution among different businesses applying or using the platform, the advantages anticipated, and relevant issues faced by the companies who have implemented or are still in the process of execution. Interviews with administration, site info, news releases in the ES system sellers, and the executing organizations depict that lots of big organizations are already utilizing ERP systems provided by top ERP 
vendors such as Oracle, SAP and AX which are being preserved by their regional partners. Several organizations will also be utilizing ERP techniques developed, executed, and maintained by local software companies. Presently a vast selection of industries big, moderate and small businesses is employing ERP systems including subsidiaries of a large multinational company, neighborhood group of organizations, textiles businesses, government and semi-government businesses, leasing firms, telecommunication businesses, tourism businesses, knit and clothing sectors.

Sometimes it's been noticed that lots of businesses have implemented their ERP applications without properly thinking about the viability of its usage to the business, in which customized applications would be sufficient as opposed to the integrated ERP systems. This was observed by Davenport as a significant aspect to consider for effective execution. 'When the business rushes to put in a business system without companies having a very crystal thorough comprehension of the company consequences, the fantasy of integration could quickly become a nightmare. The logic behind this system may struggle with logic about the company, and the execution will collapse wasting huge quantities of cash causing a lot of disturbance and also the machine will weaken significant sources of competitive benefits, hobbling the provider.' In certain massive scale associations i.e. group of businesses, the direction has a lack of knowledge about the machine and they're not even familiar with the specific function that it will provide for their own company.

That's why they have opted to purchase it without contemplating the company procedure and demands first, and in a lot of the circumstances, the execution procedure runs without appropriate preparation and therefore much less efficient as it might be. There's not any obvious thought in their mind that this system can enable them to attain their strategic aim for their company for a competitive edge. As revealed in the table of critical success factors by Finney and Corbett, best management participation is just one of the very repeatedly mentioned factors for effective execution. But an important amount of management folks thinks of it as a technological artifact as opposed to a management instrument for attaining competitive edge, to be dealt with by just the IT employees in their own company.

Many correspondences/interviews with directors disclose that in their decision-making process they haven't considered many significant critical success factors correctly. A number of them don't have any specific idea once the project is going to be completed after three decades since the execution began. Oftentimes their investors said their software investment is currently over budget and suffers from a shortage of sufficient training of their workers that are utilizing the system over a day to day basis.

Additionally, from the stakeholders' views, in several scenarios, the perceived advantages as promised by ERP vendors are not yet been achieved. Therefore, as a result of the absence of suitable comprehension of the essential factors for ERP implementation, the gap between the perceived advantages and fact is rising. However, it ought to be kept in mind that a copy cat strategy of a western model of execution won't be useful on account of the contextual topics like economic development, company environment, civilization, IT adulthood, along with telecom infrastructure, etc., that is discussed in the subsequent paragraph. Careful consideration of the problems is also a large challenge for proper execution in a developing country like Bangladesh.

\subsection{ERP Implementation Challenges in Bangladesh}

Developed countries such as the United States, the United Kingdom, and Australia do not appear to have any noticeable variations in infrastructure for individuals from the developing nations. As a consequence of infrastructural facilities such as increased IT maturity and a more favorable computer-based environment, these states may ensure that organizations operating in these settings are equipped to deal with complex technology. States in North America and Europe have remarkable infrastructures that effectively mitigate the spread of information technology from an environmental and national perspective. The desire for innovative technology is fueled even further by a strong financial basis and expansion. Technology development is fueled by government information technology policy, deregulation, and organizational enthusiasm for information technology. On the other hand, according to Rajapakse and Seddon (2005), the reasons for non-adoption in underdeveloped countries include "comparative national per capita income, as well as constrained federal infrastructure." Issues such as a lack of ability to properly deploy ERP systems and a lack of communication infrastructure limit the ability of enterprises in these states to execute these procedures in an effective and efficient manner. When compared to the developing nations of South East Asia, such issues are a reality in the case of ERP installation in Bangladesh, which is quite comparable. When it comes to developing countries, ERP technology confronts additional obstacles that are connected to cultural, economic, and core infrastructural issues.

According to them, the country's rich economic history provides a good foundation for IT/IS growth, in addition to ERP deployment. The essential prerequisite for ERP deployment is infrastructure, which includes both 
physical and information technology infrastructure. In order to support comprehensive value chain control enabled by ERP, it is critical that the entire infrastructure is in good condition. Bangladesh is experiencing infrastructure problems. Furthermore, a lack of IT maturity and a lack of a business intelligence plan make it more difficult to deploy ERP.

Most firms have a limited understanding of global business training. The amount of IT maturity could considerably influence a company's strategic decision in obtaining and deploying IT/IS. IT older organizations having a greater comprehension of IS execution can collaborate efficiently with ERP sellers, and also, therefore, are more inclined to be successful in ERP implementation. As stated previously, related to the dimensions of companies, ERP is used by big, medium, and tiny organizations. Besides, smaller businesses have started to utilize ERP because of two factors.

First and first, ERP providers are investing more effort into small and mid-sized enterprises, and secondly, small firms are feeling the need to use ERP to gain a competitive edge. Because ERP is intended to serve the whole company, business process reengineering (BPR) is usually necessary prior to ERP implementation. A company with a greater depth of experience in process management and business process reengineering (BPR) is considerably more likely to succeed with ERP. In Bangladesh, the absence of appropriate management engagement and ongoing support continues to be a stumbling block to successful implementation. Given the complexity and resource requirements, management commitment is essential to the successful deployment of ERP in both emerging and developed countries. However, considering the fundamental role of ERP in developing countries, it is possible that it will be of greater relevance in these countries.

In comparison with developed nations, the societal and organizational principles make execution of new business processes which are connected with ERP package harder in developing countries, also regarding the local division of multinational businesses. Many researchers such as Huang and Palvia, Rajapakse and Seddon, Marcia and Whitley, Thavapragasam have believed civilization is among the essential factors impacting the implementation of ERP in different nations. In their study, they utilize Hofstede's work on the federal cultural measurement of translating people's behavior in their ecological preferences on the grounds of four federal cultures. Rajapakse and Seddon (2005) asserted that the gap between energy space and the individualism/collectivism dimension can cause difficulties in the execution of western established ERP systems in Asian nations. Hofstede's cross-cultural research on Bangladesh also revealed that Bangladeshi culture is mainly hierarchical using the distinctive cultural measurement of collectivism but restricted to classes together with higher uncertainty avoidance and a greater amount of energy space. Thus, an immediate replication of western established implementation wouldn't operate in Bangladeshi culture.

\subsection{Findings that Work as a Barrier to ERP Implementation in Bangladesh}

a. Lack of skilled persons in the root label which arises the vital problem for implementing ERP.

b. Every day lot of problem arise while using this ERP system because its UI(user interface) is not so convenient for the users.

c. A lot of logistic supports are required before using this ERP, but in most of the cases it was absent for the users. That's why the total system is segregated. One department can't access other departments' data base without permission of the authorized person.

d. Sometimes it's seen that manual processing is interrupted because of incomplete system functions.

e. Every department needs to clear their work in this ERP otherwise one department's failure directly affects another department.

f. Small support team to give technical help.

g. Absence of regular training.

h. Risk of system failure.

\subsection{Recommendations}

Here are some recommendations to make the ERP implementation more convenient.

a. There is a necessity of regular training to all employees who are working with this system. It will help them to think from the core of the system and reduce the rate of making fault.

b. Awareness should be developed among all users about the importance of ERP so that everybody can realize its importance.

c. A strong support team should be established for on time trouble shooting. 
d. Monthly meeting should be arranged with all departments so that they can exchange their view of understanding and problems they have faced.

e. As Microsoft gives option to customize the system, firms need to make it more convenient as per user advices.

f. It's not a good idea to centralize the authority. It's better to create expertise in every department so that root level can get more swift support which will expedite the performance.

g. There is needed more follow up from the top management. They can check the rate of error in every month and try to reduce it by time. There should also be a discussion on common problems that are arising regularly.

\subsection{Conclusion}

It's really challenging to implement the ERP in our country. But we are hopeful that already a lot of companies have implemented the ERP system in their business. As soon as organizations can understand the advantage, it will give them a boost in their business. In far future a company or group of companies without the ERP set up can hardly be imagined. In Bangladesh some of the software firms develop their ERP system which already gets popularity in domestic market. Hope this sector will go far which can encourage our business organizations to adopt ERP.

\section{References}

Argote, L., McEvily, B., \&Reagans, R. (2003). Managing Knowledge in Organizations: An Integrative Framework and Review of Emerging Themes. Management Science,49(4), 571-582.

Bagranoff, N. A. \& Brewer, P. C. (2003). PMB investments: An enterprise system implementation. Journal of Information Systems (Spring), 85-106.

Ball,K, S. (2001). The Use of Human Resource Information Systems: a Survey. Personnel Review. International Journal of HRIS, 30(6), 667-693.

Hunton, J. E., Lippincott, B., \& Reck, J. L. (2003). Enterprise resource planning systems: comparing firm performance of adopters and non-adopters. International Journal of Accounting Information Systems, 4(3), $165-184$.

Kabir, M. R. (2020). Impact of ERP Implementation on Productivity and Profitability: An Empirical Study on the Largest Bangladeshi Steel Manufacturer. International Journal of Entrepreneurial Research, 3(4), 88-94.

Kallunki, J. P., Laitinen, E. K., \& Silvola, H. (2011). Impact of enterprise resource planning systems on management control systems and firm performance. International Journal of Accounting Information Systems, 12(1), 20-39.

Kopáčková, H., \& Škrobáčková, M. (2006) Decision Support Systems Or Business Intelligence: What Can Help In Decision Making? Scientific Papers of the University of Pardubice. Series D. Faculty of Economics and Administration, 10, 98-103.

Kovach, K. A., Hughes, A. A., Fagan, P., \& Maggitti, P. G. (2002) Administrative and Strategic Advantages of HRIS. Employment Relations Today, 29(2), 43-48. https://doi.org/10.1002/ert.10039

Nowduri, S. (2003) Management information systems and business decision making: review, analysis, and recommendations. Journal of Management and Marketing Research. Retrieved fromhttps://www.aabri.com/manuscripts/10736.pdf

Olhager, J., \& Selldin, E. (2003). Enterprise resource planning survey of Swedish manufacturing firms, European Journal of Operational Research, 146(2), 365-373. http://dx.doi.org/10.1016/S0377-2217(02)00555-6

Perry, E. (2010). The benefits of using technology in human resource management. IGI global. Cranfield School of Management.

Poston, R., \& Grabski, S. (2001). Financial impacts of enterprise resource planning implementations. International Journal of Accounting Information Systems, 2(4), 271-294.

Rajapakse, J., \& Seddon, P. B. (2005). ERP Adoption in Developing Countries in Asia: A Cultural Misfit.28th Information Systems Seminar in Scandinavia (pp. 6-9).

Rao, C. M., \& Rao, K. P. (2009). Inventory turnover ratio as a supply chain performance measure. Serbian Journal of Management, 4(1), 41-50. 
Saharia, A., Koch, B., \& Tucker, R. (2008). ERP systems and internal audit. Issues in Information Systems, 9(2), 578-586.

Statistics Finland (2011). Population Structure 2011, $\quad$ Retrieved from https://www.stat.fi/til/vaerak/2011/vaerak_2011_2012-03-16_tie_001_en.html

Dania, M. B. (2015). Enterprise Resource Planning (ERP) Systems: Design, Trends and Deployment. The International Technology Management Review, 5(2), 72-81

AlBar, D. A. M. (2014). Enterprise Resource Planning (ERP) Systems: Emergence, Importance and Challenges. The International Technology Management Review, 4(4), 170-175.

Rahad, K. A. (2014). A New Initiative for ERP System Architecture with Mobile Cloud Aspects of Bangladesh, International Conference on Electrical Engineering and Information \& communication Technology (ICEEICT).

\section{Copyrights}

Copyright for this article is retained by the author(s), with first publication rights granted to the journal.

This is an open-access article distributed under the terms and conditions of the Creative Commons Attribution license (http://creativecommons.org/licenses/by/4.0/). 